\title{
Confusion and slurred speech in a 34-year-old woman from India
}

\author{
Chandril Chugh ${ }^{1}$, N. Wadhwani ${ }^{2}$ and José Biller ${ }^{1 *}$ \\ 1 Department of Neurology, Stritch School of Medicine, Loyola University Chicago, Maywood, IL, USA \\ 2 Department of Pathology, Stritch School of Medicine, Loyola University Chicago, Maywood, IL, USA
}

Edited by:

Osvaldo Fustinoni, Universidad de

Buenos Aires, Argentina

\section{Reviewed by:}

Osvaldo Fustinoni, Universidad de

Buenos Aires, Argentina

Fernando Goldenberg, University of

Chicago Medical Center, USA

\section{*Correspondence:}

José Biller, Department of Neurology,

Stritch School of Medicine, Loyola

University Chicago, 2160 South First

Avenue, Maywood, IL 60153, USA.

e-mail: jbiller@lumc.edu
A 34-year-old woman from India, presented with episodes of confusion and progressive speech deterioration. She also had a painless neck "lump", which persisted despite antibiotics. She was diagnosed to have a space occupying lesion in the left parietal lobe, which was subsequently biopsied.

Keywords: brain mass lesion, cerebral edema, speech deterioration, neck mass
A 34-year-old woman born in India, presented to our clinic with concerns of confusion and slurred speech. Three months before presentation, she had occasional episodes of confusion. During these episodes, she was unable to respond, but never lost consciousness. These events were brief and resolved completely. She worked at a grocery store with her husband, who noticed she was having trouble tending right change to the customers. She also had difficulty remembering names of the employees. Two months earlier she started slurring and even her husband had difficulty understanding her. She then had a generalized tonic clonic seizure. She also complained of lethargy and body aches accompanied by a low grade fever. She did not have any weight loss or night sweats.

She was last seen by a physician a year earlier, for a "neck lump." She was started on a course of antibiotics, but the lump persisted. She did not have a history of smoking, alcohol abuse, or intravenous drug abuse. There was no past history of hypertension, diabetes mellitus, cardiac arrhythmias, or stroke. Her father had a history of hypertension and pulmonary tuberculosis, but she was not living with him for the past 10 years.

She had an unremarkable physical exam, except for a left sided soft, non-tender, fluctuant, mobile neck mass, measuring around $3 \mathrm{~cm} \times 5 \mathrm{~cm}$ in size. Neurological examination showed deficits in attention and concentration. She had difficulty performing simple calculations. Speech was slurred but language was intact. She did not have any cranial nerve deficits, motor weakness, or sensory deficits. She had a normal gait and stance.

Laboratory testing showed normal blood indices. She had a mildly elevated erythrocyte sedimentation rate. HIV antibody testing was negative. Chest radiograph was unremarkable. She had an aspiration biopsy of the neck mass, which showed acid fast organisms on Ziehl-Neelsen staining. Cranial computerized tomography (CT) showed a left parietal lobe mass lesion with surrounding edema. She had resection of the mass lesion.
The patient is currently being followed in the neurology outpatient clinic and has improved since the surgery and institution of antitubercular drug therapy. The residual neck scar is shown in Figure 1 below.

\section{PATHOLOGY}

Histology sections revealed multiple foci of caseating granulomas with central necrosis immediately surrounded by a dense rim of macrophages and a rim of lymphocytes, Figures 2 and 3. ZiehlNeelsen staining shows a single rod shaped, acid fast organism, Figure 4.

\section{DISCUSSION}

Tuberculosis is the world's leading cause of death due to a single infectious agent. The tuberculosis bacterium was discovered by Koch in 1882, but the description of the disease dates back to the times of Hippocrates and Galen (MacGregor, 1993). Tuberculosis is more prevalent in the developing countries as compared to developed countries. Since the discovery of streptomycin in 1946, the mortality rate due to tuberculosis has steadily declined, but due to HIV epidemic, the world is seeing a resurgence of this disease. New multidrug resistant strains have emerged and pose a serious threat to the health of people exposed to it.

Tuberculosis of the central nervous system (CNS) is a well known entity. It was described first by Whytt in 1768, in a patient with hydrocephalus and febrile illness (MacGregor, 1993). Tuberculosis can involve the nervous system in a number of ways like, meningitis, brain abscess, cerebral venous sinus thrombosis, transverse myelitis, and hydrocephalus.

Mycobacterium tuberculosis is an airborne microorganism. The lungs are usually the primary site of infection. However the kidneys, lymph nodes, spine, and CNS may be infected first. The CNS may get involved due to dissemination of infection through the lymphohematogenous route from other primary sites (Rich 


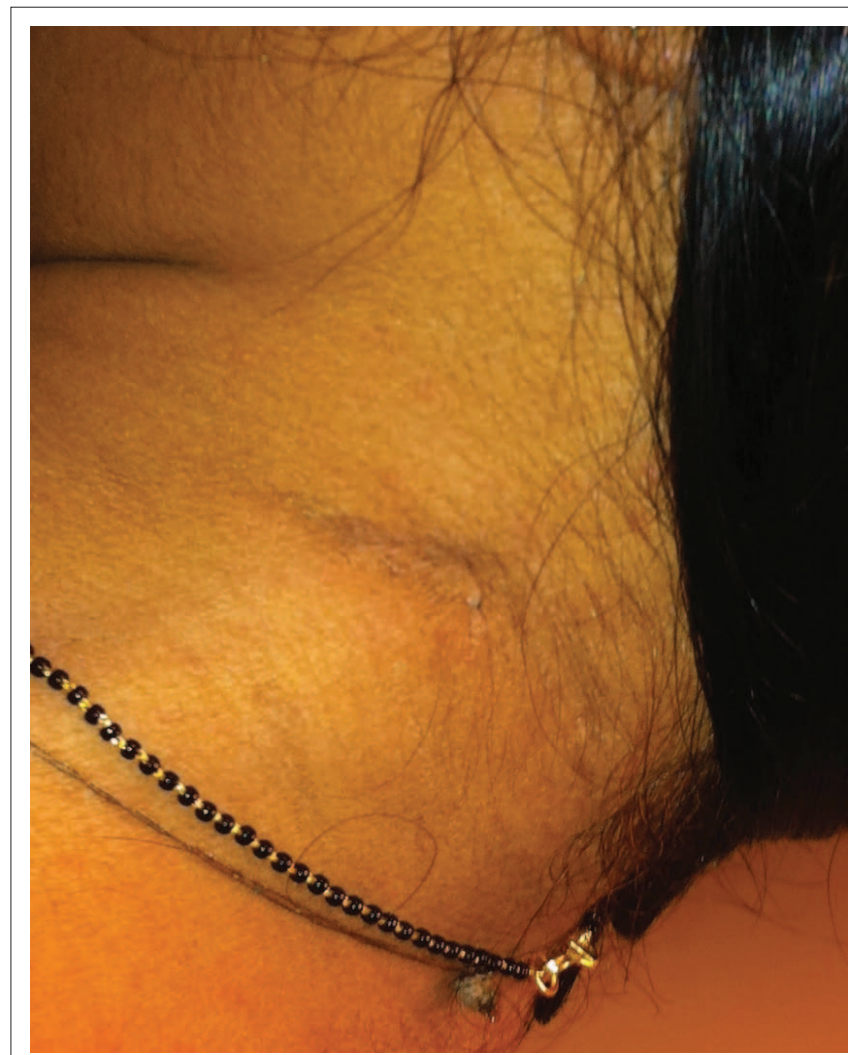

FIGURE 1 | Ziehl-Neelsen staining.

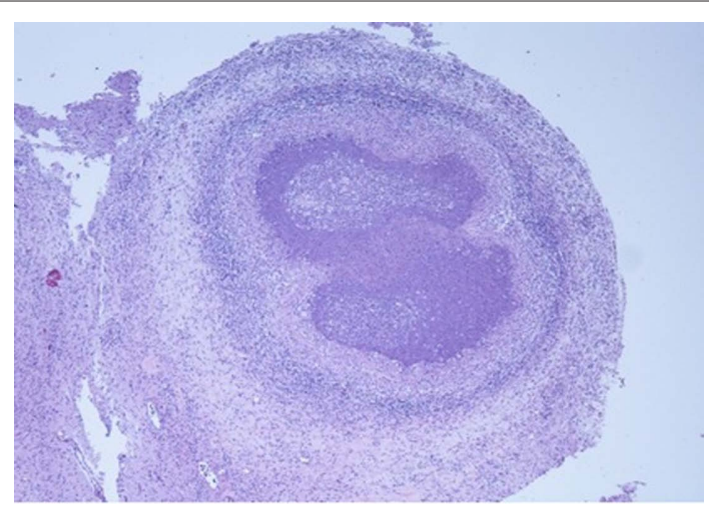

FIGURE 2 | Hematoxylin and eosin staining.

and McCordick, 1933). The most common site of extrapulmonary tuberculosis is cervical lymph nodes but inguinal, axillary, mesenteric, mediastinal, and intramammary lymph node involvement have been seen (Artenstein et al., 1995). Tuberculous lymphadenitis has a peak age of onset of 20-40 years, and in the United States it is most common in women and among immigrants from high risk areas. Patients without HIV infection typically present with chronic, non-tender lymphadenopathy (Jha et al., 2001; Marjorie et al., 2005).

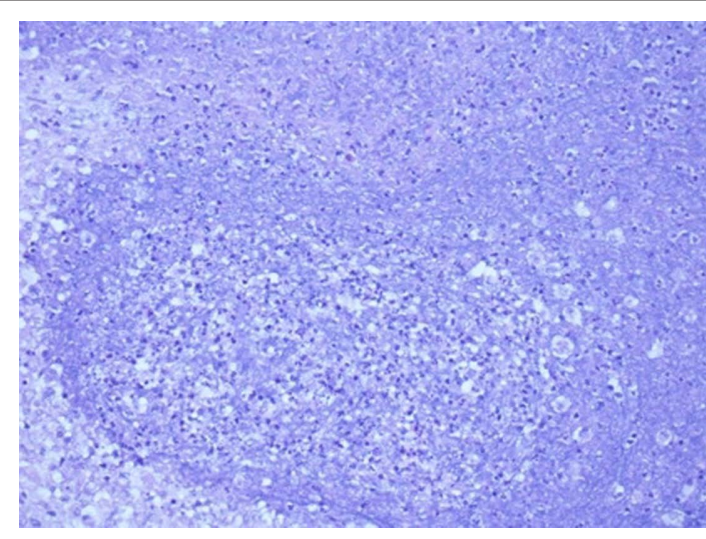

FIGURE 3 | Hematoxylin and eosin staining.

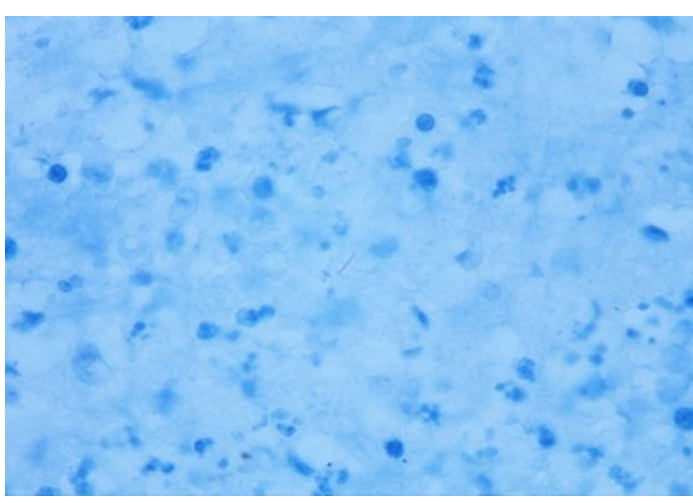

FIGURE 4 | Hematoxylin and eosin staining.

Tubercular meningitis is the most common presentation of nervous system tuberculosis. Tuberculoma of the brain, on the other hand, is rare. It is usually formed by conglomeration of several miliary tubercles, which form around the outer sheaths of the cerebral blood vessels. Caseous necrosis ensues and the necrotic debris gets inspissated and sometimes liquefied. A thick capsule may form around these lesions. The incidence of tuberculoma among all intracranial neoplasm ranges from $0.2 \%$ in the industrialized world to around $8 \%$ in the developing world (Bouchama et al., 1991; Abdul-Ghaffar et al., 1998; Artico et al., 1999).In North America the incidence of tuberculoma ranges from $0.7 \%$ to $1.4 \%$ (Sibley and O'Brien, 1956).

A tuberculoma is silent till neurological symptoms appear due to mass effect or when it communicates with the subarachnoid space causing meningitis (Bishburg et al., 1986). Patients usually present with malaise, low grade fever, headaches, confusion, or weight loss. Confusion in the absence of fever may be seen. History of previous tuberculosis infection may be present in only half of the cases (Kent et al., 1993). Depending on the site of infection the patient may present with cranial nerve palsies (Traub et al., 1984), visual impairment (Teoh and Humphries, 1991), hemiparesis, ataxia, tremor, or seizures. Movement disorders like chorea, hemiballismus, myoclonus, and athetosis may also be seen (Alarcon et al., 2000). Patients with HIV infection are at a higher 
risk of tuberculosis, however there is controversial evidence if tuberculomas are seen with increased frequency in these patients (Whiteman, 1997). Tuberculomas are most commonly seen in frontal lobe, cerebellum, and brainstem.

Diagnosis of tuberculoma is confirmed by imaging followed by biopsy or excision of the lesion. Presence of epithelioid cells and granulomatous inflammation, differentiate tuberculoma from a tubercular brain abscess. CT or magnetic resonance imaging of the head may show meningeal enhancement, hydrocephalus, edema, or infarction, with a space occupying lesion (Draouat et al., 1987). Tuberculomas in younger patients are generally solitary (80\%) and appear isodense or hypodense areas with uniform enhancement. In later stages ring enhancement is seen (Kumar et al., 1993).In the early stages, tuberculoma may be confused with a low grade astrocytoma. Later on, when there is a uniform enhancement, other differentials, such as sarcoid granulomas, mycotic granulomas, astrocytomas, lymphoma, toxoplasma, and metastasis should be considered.

The management of tuberculomas due to Mycobacterium tuberculosis includes institution of antitubercular drug regimen. Current regimen includes four drugs (isoniazid, rifampicin, pyrazinamide,

and ethambutol) for 2 months followed by two drugs (isoniazid and rifampicin) for at least 10 months (Thwaites et al., 2009). Treatment of Mycobacterium avium intracellulare constitutes azithromycin, clarithromycin with ethambutol or clofazimine (Kemper et al., 1992). In patients with HIV infection side effects may be encountered in the first few months of the treatment. In such cases changing the drug regimen may be helpful (Small et al., 1991). Surgery may be needed for large tuberculomas with mass effect and when diagnosis of tuberculosis is in doubt. Surgery has also been tried in patients not responding to treatment ( $\mathrm{Li}$ et al., 2006).

Despite aggressive management and surgical intervention the survival of patients with CNS tuberculosis is not good. In a number of studies very high mortality rates of up to $50 \%$ have been reported (Porkert et al., 1997). Neurological sequelae vary with age and demographics. Commonly, seizures, hemiparesis, ataxia, or cranial nerve palsies may be seen in survivors of tubercular infection (Ogawa et al., 1987). In children cognitive and motor impairment may be seen and may not improve after treatment (Schoeman et al., 1997).

Tuberculous meningitis in an urban medical center. Medicine (Baltimore) 66, 317-326.

Porkert, M. T., Blumberg, H. M., ParrottMoore, P., and Sotir, M. (1997). Tuberculous meningitis at a large inner-city medical center. Am. J. Med. Sci. 313, 325-331.

Rich, A. R., and McCordick, H. A. (1933). The pathogenesis of tuberculous meningitis. Bull. Johns Hopkins Hosp. 52, 5-37.

Schoeman, C. J., Herbst, I., and Nienkemper, D. C. (1997). The effect of tuberculous meningitis on the cognitive and motor development of children. S. Afr. Med. J. 87, 70-72.

Sibley, W. A., and O'Brien, J. L. (1956). Intracranial tuberculomas: a review of clinical features and treatment. Neurology 6, 157-165.

Small, P. M., Schecter, G. F., Goodman, P. C., Sande, M. A., Chaisson, R. E., and Hopewell, P.C. (1991). Treatment of tuberculosis in patients with advanced human immunodeficiency virus infection. N. Engl. J. Med. 324, 289-294.

Teoh, R., and Humphries, M. (1991). "Tuberculous meningitis," in Infections of the Central Nervous System, ed. H. P.Lambert (Philadelphia: BC Decker), 189-206.

Thwaites, G., Fisher, M., Hemingway, C., Scott, G., Solomon, T., and Innes, J. (2009). British Infection Society guidelines for the diagnosis and treat- ment of tuberculosis of the central nervous system in adults and children. J. Infect. 59, 167-187.

Traub, M., Colchester, A. C., Kingsley, D. P., and Swash, M. (1984). Tuberculosis of the central nervous system. Q. J. Med. 53, 81-100.

Whiteman,M.L. (1997). Neuroimaging of central nervous system tuberculosis in HIV-infected patients. Neuroimaging Clin. N. Am. 7, 199-214.

Conflict of Interest Statement: The authors declare that the research was conducted in the absence of any commercial or financial relationships that could be construed as a potential conflict of interest.

Received: 12 March 2011; accepted: 10 May 2011; published online: 06 June 2011. Citation: Chugh C, Wadhwani $N$ and Biller J (2011) Confusion and slurred speech in a 34-year-old woman from India. Front. Neur. 2:34. doi: 10.3389/ fneur.2011.00034

This article was submitted to Frontiers in Neurology Education, a specialty of Frontiers in Neurology.

Copyright (ㅇ 2011 Chugh, Wadhwani and Biller. This is an open-access article subject to a non-exclusive license between the authors and Frontiers Media SA, which permits use, distribution and reproduction in other forums, provided the original authors and source are credited and other Frontiers conditions are complied with. 